\title{
Dietary conjugated linoleic acid (CLA) intake assessment and possible biomarkers of CLA intake in young women
}

\author{
Dorothee Fremann ${ }^{1, *}$, Jakob Linseisen ${ }^{1,2}$ and Günther Wolfram ${ }^{1}$ \\ 'Lehrstuhl für Ernährungslehre, Department für Lebensmittel und Ernährung, Technische Universität München- \\ Weihenstephan, Alte Akademie 16, D-85350 Freising, Germany: ${ }^{2}$ Deutsches Krebsforschungszentrum, \\ Abteilung für Klinische Epidemiologie, Im Nevenheimer Feld 280, D-69120 Heidelberg, Germany
}

Submitted 29 January 2001: Accepted 15 June 2001

\begin{abstract}
Objective: The habitual intake of the conjugated linoleic acid (CLA) isomer C18:2 c9t11 (rumenic acid, RA) was assessed and compared with plasma biomarkers.

Design: The newly developed food-frequency questionnaire (FFQ) comprised 46 food items and was validated by means of a 7-day estimated record (7-d ER). Additionally, the dietary intake results of the FFQ, 7-d ER, the last day (1-d ER) and the last two days (2-d ER) before blood sampling of the 7-d ER were compared to the content of C18:2 c9t11 in plasma phospholipids (PL) and triglycerides (TG) as possible biomarkers.

Setting: Metabolic unit of a university institute.

Subjects: Fifty-seven students completed both dietary instruments. From all participants fasting blood samples were taken.

Results: Mean daily intake of rumenic acid was $246 \mathrm{mg} \mathrm{day}^{-1}$ and $323 \mathrm{mg} \mathrm{day}^{-1}$ as measured by the FFQ and the 7-d-ER, respectively. The degree of correspondence between both assessment methods was acceptable; this is indicated by a total kappa value of $\kappa=0.31(P<0.01)$ and a Pearson correlation coefficient of $r=0.46(P<$ 0.01). Rumenic acid content in plasma triglycerides was twice as high as found in phospholipids. The correlation between the intake results gained with the 7-d ER and the plasma PL contents of C18:2 c9t11 was statistically significant; this was also true for the C18:2 c9t11 values in plasma TG compared with the intake results of one or two days before blood sampling.

Conclusions: Regarding RA intake, the FFQ data revealed an acceptable degree of correspondence with the 7-d ER data but failed to show significant correlations to the potential biomarkers. However, with respect to the results of the 7-d ER, the RA content in plasma PL and TG are possible biomarkers of short-term and mediumterm intake, respectively.
\end{abstract}

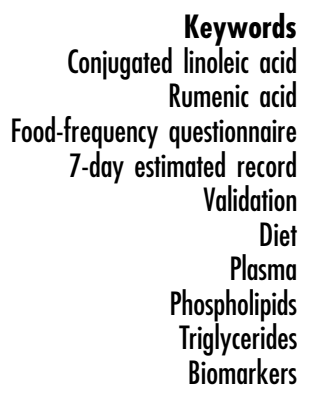

The group of the conjugated linoleic acid isomers (CLA) consists of different geometrical double-bond configurations and positional isomers of linoleic acid. The major CLA isomers identified are C18:2 t8c10, C18:2 c9t11, C18:2 t10c12 and C18:2 c11t13 ${ }^{1}$. Predominantly, the anaerobic bacterium Butyrivibrio fibrisolvens, localised in the ruminant stomach, is capable of metabolising linoleic acid (C18:2 c9c12) to CLA, mainly the C18:2 c9t11 isomer, also called rumenic acid. Therefore, in the human diet, rumenic acid is provided primarily by the intake of dairy products and meat from ruminants and represents the quantitatively most important CLA isomer ${ }^{2}$.

Due to the potential health effects demonstrated in animal and cell culture experiments, CLA has attracted much scientific attention. CLA was reported to reveal anticarcinogenic $^{3-5}$ and anti-atherogenic effects ${ }^{6,7}$ while the underlying mechanisms are still unclear. Of the different isomers of CLA, rumenic acid has been indicated as the most bioactive ${ }^{8}$. However, the CLA isomer C18:2 t10c12 may also exert profound biological effects? ${ }^{9}$. In humans, much less is known about the physiological effects of CLA isomers ${ }^{10-13}$.

Few estimations of dietary CLA intake are available ${ }^{14,15}$, and information on the bioavailability of CLA from foods or supplements is scarce ${ }^{16,17}$. Moreover, data on the extent of incorporation of dietary CLA into plasma lipids are limited in humans ${ }^{18}$. The suitability of plasma CLA contents as biomarkers of their intake has not been assessed so far.

In experimental studies dealing with the biological effects of CLA isomers, the habitual dietary exposure has to be considered. The average long-term diet is best 
measured by means of a food-frequency questionnaire (FFQ) although its limitations are obvious ${ }^{19}$. Therefore, in the present study, a short FFQ for assessment of the habitual CLA intake has been developed. To validate the new dietary assessment method, comparisons were made to the 'gold standard' of a dietary record over 7 days as well as to possible biomarkers: the CLA content in plasma lipids (as already established for other fatty acids).

\section{Subjects and methods}

\section{Subjects}

Fifty-seven female university students were enrolled in the study, all of whom met the inclusion criteria (no pregnancy, no breast-feeding, no metabolic disorders, no dieting). An experienced nutritionist instructed the study participants in the completion of the FFQ and the 7-day estimated record (7-d ER). During the 7 days of dietary recording, each student was contacted at least once by telephone. The completed 7-d ER was handed in personally, enabling a brief check of the records. The coding of the FFQ and the 7-d ER was done within the following two months, and in the case of problems (e.g. missing recipes) the participants could be re-contacted. Fasting blood samples were drawn from each participant at the end of the protocol period.

\section{Development of the food-frequency questionnaire}

The FFQ was developed to assess the usual intake of rumenic acid and total fat. Butter, milk, milk products, cheese, meat, meat products are reported to be the food items mainly contributing to the total rumenic acid intake $^{14}$. A short questionnaire designed to assess fat intake $^{20}$ (fat screener), including 20 validated food items, was taken as a basis and adapted. With inclusion of the most important CLA-providing foods, the developed FFQ included 46 food items corresponding to seven food groups: 'meat, meat products \& fish', 'fat', 'milk \& milk products', 'eggs', 'bread', 'cake \& sweets' and 'others'. The portion sizes were indicated in household measures (such as a glass of milk or one piece of cake). Sometimes, the portions were defined as small, average or large (e.g. average piece of bread). Nine consumption frequency categories are offered in the range from 'never' to 'more than 2 times per day'. Regarding the food group 'milk \& milk products', two further questions on the fat content chosen usually were inserted. The final FFQ version was handed out with a short written instruction to provide a tool that could be self-administered; however, due to the personal contact, the content of the handout was repeated orally.

The fat content of most food items in the FFQ was estimated as a weighted average. The weighting was created on the basis of calculations at the German National Food Consumption Study ${ }^{21}$. If weighting was not necessary (e.g. butter, milk), the fat content as listed in the German Federal Food Code (BLS) nutrient database (version II.2) was used. Analytical values of rumenic acid were taken mainly from German food analyses ${ }^{14}$. Food coding and calculation were done using SPSS for Windows, version 9 (SPSS, Inc., Chicago, IL).

\section{Seven-day estimated record}

The 7-d ER was selected as reference method ('gold standard'). The students were asked to record all foods and beverages in a booklet. Whenever possible, the foods should be weighed; if necessary digital scales and a measuring beaker were handed out to the students. The participants were also instructed in the use of household measures. For this purpose, they were asked to measure the content of their usual cup of coffee, glasses and bowls once at the beginning of the study. Students were encouraged to provide details of home-made recipes, including the weight of each ingredient and of the individual serving after cooking. For meals consumed in a restaurant, standard recipes were used $^{22}$.

The coding of the 7-d ER was performed by a nutritionist and controlled by another using the nutrition software Prodi 3+ (Wissenschaftliche Verlagsgesellschaft, Stuttgart, Germany) with the nutrient database Souci/ Fachmann/Kraut (1986). This program version easily allows the addition of further nutrients such as rumenic acid. The inserted analytical data of the rumenic acid content of food consisted mainly of results of German food analyses ${ }^{14}$.

To control underreporting, the basal metabolic rate (BMR) was calculated ${ }^{23}$ and compared with the reported mean daily energy intake (7-d ER). A ratio of energy intake to BMR lower than 1.1 was judged as underreporting ${ }^{24}$ and the corresponding person was excluded from the study.

\section{Blood sampling and fatty acid analysis}

Blood samples were drawn after at least $12 \mathrm{~h}$ of fasting into tubes containing ethylenediamine tetraacetic acid (EDTA). The plasma samples obtained were stored until analysis at $-80^{\circ} \mathrm{C}$.

Briefly, lipids were extracted from $1 \mathrm{ml}$ aliquots of plasma using a Folch-Sperry extract. Triglycerides (TG) and phospholipids (PL) were separated by means of thin-layer chromatography as described in detail elsewhere ${ }^{25,26}$. After transesterification with trimethylsulfonium hydroxide (TMSH) ${ }^{27}$, the fatty acid methyl esters were analysed using a Hewlett-Packard 5890 Series II gas chromatograph (GC), equipped with a flame ionisation detector and a $50 \mathrm{~m}$ fused silica capillary column (CP-Sil 88, Varian-Chrompack GmbH, Darmstadt, Germany). The fatty acids were identified by means of the retention time of standard substances (Sigma, Deisenhofen, Germany; Matreya, Biotrend, Köln, Germany). For identification of rumenic acid, two commercially available standards were used to distinguish between the CLA isomers C18:2 c9t11 
Table 1 Characteristics of the 52 subjects included in the validation study

\begin{tabular}{lccc}
\hline & Mean \pm SD & Median & Range \\
\hline Age (years) & $24.19 \pm 2.62$ & 24 & $22-36$ \\
Weight $(\mathrm{kg})$ & $61.37 \pm 10.22$ & 58.05 & $44.80-95.90$ \\
Height $(\mathrm{m})$ & $1.69 \pm 0.06$ & 1.68 & $1.54-1.83$ \\
Body mass index, BMI $\left(\mathrm{kg} \mathrm{m}^{-2}\right)$ & $21.52 \pm 2.83$ & 20.90 & $17.18-29.93$ \\
BMR (kcal day $\left.{ }^{-1}\right) \dagger$ & $1423.57 \pm 103.6$ & 1398.05 & $1204.90-1761.10$ \\
\hline
\end{tabular}

† Harris-Benedict formula.

and C18:2 t10c12/c10t12. Additionally, fatty acid analysis of food samples of ruminant origin showed a peak with the retention time of the C18:2 c9t11 standard. Although minor CLA isomers might be included in the C18:2 c9t11 peak area, it seems obvious that the major compound by far is represented by C18:2 c9t11. Since this study did not aim at getting information on the physiological effects of different CLA isomers, it was determined unnecessary to apply a more exact but much more laborious analytical method to get a more complete resolution of the different CLA isomers in the GC spectra.

\section{Statistical analysis}

Descriptive statistics are given in terms of mean, standard deviation (SD), median, range and percentiles. All calculations were performed using SPSS for Windows, version 9 (SPSS, Inc., Chicago, IL). To test for statistically significant differences between the results gained by FFQ and 7-d-ER, the Wilcoxon test was applied.

To analyse the degree of correspondence of the results between both dietary methods, a cross-classification using tertiles was performed followed by calculation of the kappa statistic ${ }^{28}$. The kappa value is an indicator of the degree of the corresponding ranking of subjects by the two different methods. Additionally, the Pearson correlation coefficient was calculated to examine the degree of correspondence between both dietary assessment methods as well as between intake data and plasma fatty acid concentrations.

\section{Results}

From the 57 students enrolled, five were excluded. Three showed distinct underreporting, the 7-d ER of one student could not be analysed (high amount of Asiatic food), and the FFQ of one student was lacking most answers. The characteristics of the remaining 52 female participants are given in Table 1.

The mean daily intake of rumenic acid was $246 \mathrm{mg}$ day $^{-1}$ and $323 \mathrm{mg} \mathrm{day}^{-1}$ as measured by the FFQ and the 7-d-ER, respectively; the mean total fat intake was $63 \mathrm{~g}$ day $^{-1}$ and $82 \mathrm{~g} \mathrm{day}^{-1}$, respectively (Table 2). The differences between both assessment methods were statistically significant. Consequently, the FFQ revealed mean intake values 24\% (rumenic acid) and 23\% (total fat) lower than found with the 7-d ER. The between-person

Table 2 Dietary intake of CLA isomer C18:2 c9t11 (mg day ${ }^{-1}, \% \mathrm{FA} \dagger, \%$ ent) and total fat $\left(\mathrm{g} \mathrm{day}{ }^{-1}\right)(\mathrm{mean} \pm \mathrm{SD}$, median, range, percentiles) as assessed by the developed food-frequency questionnaire (FFQ) and the 7-day estimated record (7-d ER) ( $n=52)$. The results of the last day (1-d ER) and the last two days (2-d ER) before blood sampling of the 7-d ER are given separately

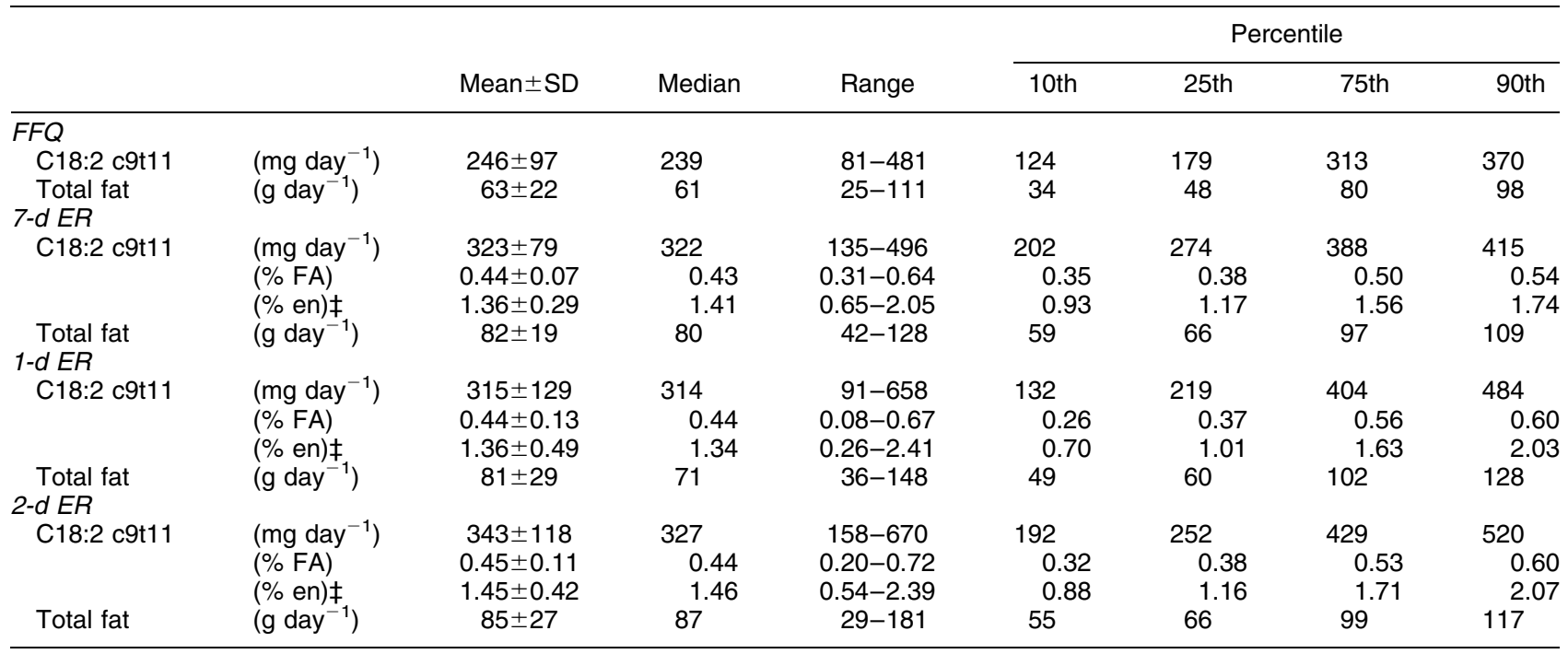

$\dagger \% \mathrm{FA}$ - percentage of total fatty acid intake; \% en - percentage of total energy intake.

$\ddagger \times 10^{-3}$. 
Table 3 Agreement of the results of rumenic acid (C18:2 c9t11) and total fat obtained by means of both dietary instruments, the newly developed food-frequency questionnaire (FFQ) and the 7-day estimated record (7-d ER): classification into tertiles with values of the kappa statistic and Pearson correlation coefficient

\begin{tabular}{lcc}
\hline & $\begin{array}{c}\text { C18:2 c9t11 } \\
\left(\mathrm{mg} \mathrm{day}^{-1}\right)\end{array}$ & $\begin{array}{c}\text { Total fat } \\
\left.\text { (g day }^{-1}\right)\end{array}$ \\
\hline $\begin{array}{l}\text { Classification in tertiles } \\
\quad \text { Same tertile (\%) }\end{array}$ & 54 & 42 \\
$\quad$ Adjacent tertile (\%) & 35 & 48 \\
$\quad$ Opposite tertiles (\%) & 11 & 10 \\
Kappa statistic & & \\
1. tertile & 0.22 & 0.25 \\
2. tertile & 0.24 & 0.05 \\
3. tertile & 0.47 & 0.13 \\
$\quad$ Total & $0.31^{*}$ & 0.13 \\
Pearson's correlation coefficient & $0.46^{*}$ & $0.43^{*}$ \\
\hline * $P<0.01$ & &
\end{tabular}

variability as measured by standard deviation was higher in the FFQ than the corresponding values given by the 7-d ER. The distribution of foods that provided rumenic acid was determined using data collected by 7-d ER. Using 7-d ER data, milk \& milk products and butter were the primary sources of rumenic acid, contributing 59\% and $28 \%$ of total RA intake, followed by meat $\&$ meat products and others.

The results of the measures used to characterise the agreement of both dietary assessment methods to obtain rumenic acid and total fat intake data are summarised in Table 3. The majority of the students were in the same tertile of rumenic acid intake. On the other hand, more subjects were in adjacent tertiles of total fat intake than in the same tertile. Resulting total kappa values were 0.31 and 0.13 for rumenic acid and total fat intake, respectively. A kappa value of 0.31 can be interpreted as an indicator of 'middle agreement' and a kappa value of 0.13 means 'bad agreement' of both assessment methods ${ }^{29}$. The calculated correlation coefficients between both methods of data collection were statistically significant for rumenic acid as well as for total fat intake. The regression line as depicted in Fig. 1 confirms the significant linear association between both assessment methods for dietary rumenic acid intake.

The results of the fatty acid analysis in plasma PL and TG demonstrates that the mean proportion of rumenic acid was about twice as high in TG than in PL (Table 4). However, the range of rumenic acid contents in plasma TG was much higher than found in plasma PL.

Table 5 shows the association between rumenic acid intake and its proportion in plasma PL and TG fatty acids. The correlation between intake, calculated from the FFQ, and the proportions found in plasma PL and TG was poor. In contrast, the average intake values of rumenic acid (percentage of fatty acids, \% FA) calculated by means of the 7-d ER correlated significantly with its content in plasma PL (percentage of fatty acid methyl esters, \% FAME). The corresponding correlation coefficients were in the range of $0.28-0.36$. The strongest relationship existed between the intake of rumenic acid (\% FA) calculated from the last day (1-d ER) or the last two days (2-d ER) before blood sampling of the 7-d ER and the

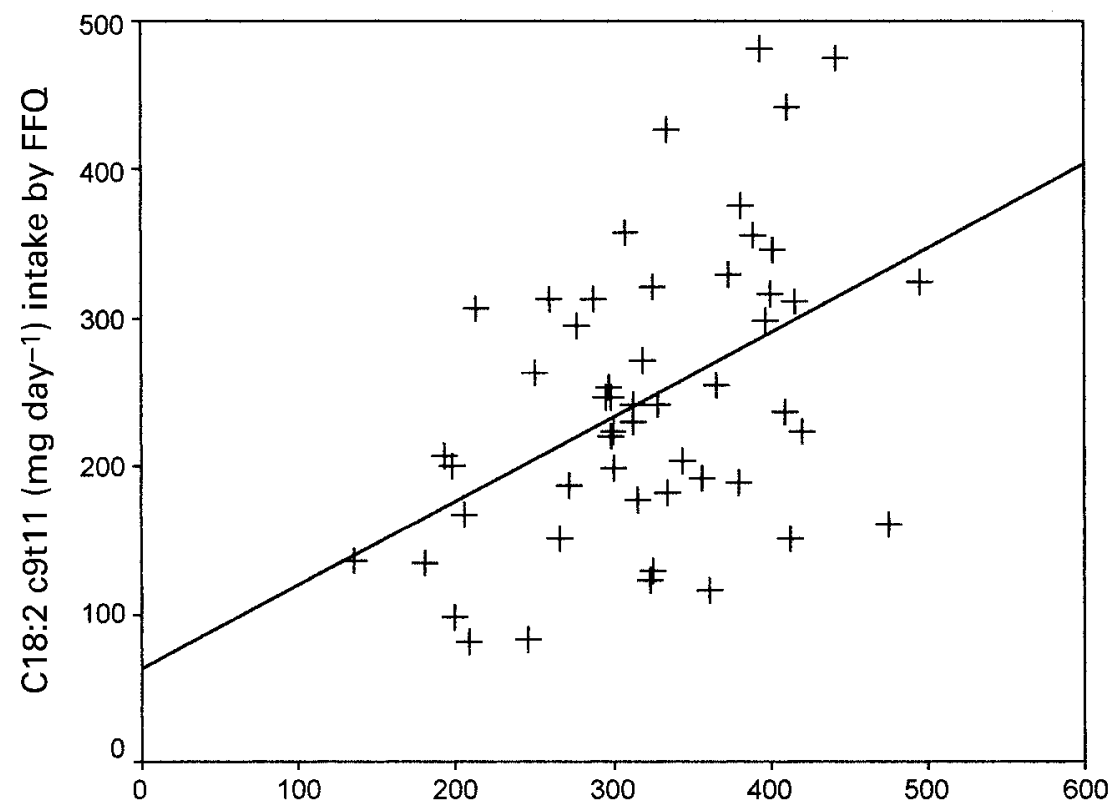

C18:2 c9t11 (mg day ${ }^{-1}$ ) intake by 7-d ER

Fig. 1 Scatter plot and regression line $\left(y=62.5+0.57 x ; r^{2}=0.215 ; P<0.01\right)$ for dietary rumenic acid intake as assessed by the developed food-frequency questionnaire (FFQ) and the 7-day estimated record (7-d ER) 
Table 4 Fatty acid composition (\% FAME†) of plasma phospholipids and triglycerides in 52 female university students

\begin{tabular}{|c|c|c|c|c|c|c|c|}
\hline \multirow[b]{2}{*}{ Fatty acid } & \multirow[b]{2}{*}{ Mean $\pm S D$} & \multirow[b]{2}{*}{ Median } & \multirow[b]{2}{*}{ Range } & \multicolumn{4}{|c|}{ Percentile } \\
\hline & & & & 10th & 25 th & 75th & 90th \\
\hline \multicolumn{8}{|l|}{ Phospholipids } \\
\hline C18:2 c9t11 & $0.27 \pm 0.01$ & 0.26 & $0.11-0.49$ & 0.17 & 0.21 & 0.32 & 0.38 \\
\hline $\mathrm{C} 16: 0$ & $29.89 \pm 2.04$ & 29.89 & $25.57-34.67$ & 27.27 & 28.53 & 31.70 & 32.45 \\
\hline C16:1 & $0.95 \pm 0.21$ & 0.94 & $0.52-1.42$ & 0.65 & 0.82 & 1.09 & 1.24 \\
\hline C18:0 & $13.70 \pm 1.51$ & 14.00 & $10.89-16.72$ & 11.67 & 12.34 & 14.84 & 15.54 \\
\hline C18:1 & $10.85 \pm 1.10$ & 10.85 & $8.62-13.45$ & 9.24 & 10.08 & 11.58 & 12.18 \\
\hline C18:2 & $22.48 \pm 2.34$ & 22.57 & $18.87-29.09$ & 19.51 & 20.26 & 23.75 & 25.28 \\
\hline C18:3 n3 & $0.30 \pm 0.14$ & 0.28 & $0.06-0.86$ & 0.14 & 0.20 & 0.37 & 0.49 \\
\hline C20:3 n6 & $0.01 \pm 0.01$ & 0.01 & $0.00-0.25$ & 0.00 & 0.00 & 0.01 & 0.14 \\
\hline C20:4 n6 & $10.68 \pm 1.78$ & 10.55 & $7.43-17.85$ & 8.48 & 9.58 & 11.48 & 12.83 \\
\hline C20:5 n3 & $0.75 \pm 0.38$ & 0.67 & $0.28-2.59$ & 0.44 & 0.53 & 0.87 & 1.19 \\
\hline C22:6 n3 & $4.66 \pm 1.13$ & 4.39 & $2.86-7.78$ & 3.51 & 3.85 & 5.44 & 6.20 \\
\hline \multicolumn{8}{|l|}{ Triglycerides } \\
\hline C18:2 c9t11 & $0.54 \pm 0.12$ & 0.55 & $0.33-0.97$ & 0.40 & 0.46 & 0.60 & 0.71 \\
\hline $\mathrm{C} 16: 0$ & $30.00 \pm 3.54$ & 29.88 & $21.20-39.94$ & 26.18 & 27.53 & 32.26 & 34.73 \\
\hline C16:1 & $4.76 \pm 1.14$ & 4.88 & $2.18-8.06$ & 3.37 & 3.90 & 5.35 & 5.89 \\
\hline C18:0 & $5.61 \pm 1.62$ & 5.23 & $3.03-10.06$ & 3.77 & 4.27 & 6.50 & 8.11 \\
\hline C18:1 & $36.15 \pm 3.17$ & 36.55 & $26.75-42.68$ & 32.59 & 33.70 & 38.54 & 40.16 \\
\hline C18:2 & $15.57 \pm 3.71$ & 15.03 & $10.57-32.34$ & 11.50 & 13.13 & 17.36 & 19.73 \\
\hline C18:3 n3 & $0.87 \pm 0.23$ & 0.81 & $0.44-1.43$ & 0.61 & 0.70 & 1.02 & 1.24 \\
\hline C20:3 n6 & $0.38 \pm 0.18$ & 0.37 & $0.05-0.89$ & 0.18 & 0.25 & 0.49 & 0.66 \\
\hline C20:4 n6 & $1.31 \pm 0.42$ & 1.27 & $0.63-2.48$ & 0.77 & 0.96 & 1.59 & 1.86 \\
\hline C20:5 n3 & $0.25 \pm 0.18$ & 0.19 & $0.00-0.74$ & 0.01 & 0.13 & 0.28 & 0.59 \\
\hline C22:6 n3 & $0.66 \pm 0.31$ & 0.66 & $0.13-1.88$ & 0.31 & 0.48 & 0.86 & 1.02 \\
\hline
\end{tabular}

† FAME - fatty acid methyl esters.

rumenic acid content in plasma TG (\% FA). No significant correlations were found for the intake of rumenic acid expressed as a percentage of total energy intake (calculated from 7-d ER, 1-d ER and 2-d ER; Table 2) and the proportion of rumenic acid found in plasma PL and TG (\% FAME). All correlation coefficients were below 0.2 (except for 1 -d ER versus plasma TG, with $r=0.268$ ).

\section{Discussion}

The present study was conducted to test the validity of a newly developed FFQ and to assess the relationship between rumenic acid intake and its plasma content. Due to the study design, sex and age as possible confounding variables were excluded and the study was conducted in a very homogeneous group of young women. A recognised methodology to create an FFQ food list is to modify an existing FFQ that is based on a similar target and applied in the same cultural surrounding ${ }^{19}$. The FFQ developed here was created on the basis of an already existing qualitative questionnaire comprising 20 food items to estimate the intake of total fat, polyunsaturated fatty acids, monounsaturated fatty acids, saturated fatty acids and cholesterol in Germany ${ }^{20}$. Therefore, this questionnaire represented a suitable basis for adaptation to the new task.

When comparing the mean intake of rumenic acid and total fat with both assessment methods, it becomes obvious that the FFQ provides lower values than found with the 7-d ER. In similar validation studies comparing the results of an FFQ with those of a dietary record, underestimation as well as overestimation of fat and polyunsaturated fatty acid intake by means of an FFQ were reported ${ }^{30-32}$. However, most validation studies showed a relatively good agreement of total fat and fatty acid intake results calculated by both dietary assessment methods ${ }^{33-37}$.

Results from a recent study in 46 males and 47 females showed that the intake of rumenic acid estimated by FFQ was 151 and $72 \mathrm{mg}$ day $^{-1}$ for men and women,

Table 5 Pearson's correlation coefficients for the C18:2 c9t11 content in plasma phospholipids (PL) and triglycerides (TG) (\% FAME†) and its calculated intake as assessed by the newly developed food-frequency questionnaire (FFQ, \% FA†) or by the full 7-day estimated record (7-d ER, \% FA†), the last day of the 7-d ER (1-d ER $\ddagger$, \% FAt) and the last two days of the 7-d ER (2-d ER $\neq$ \% FA†) $(n=52)$

\begin{tabular}{lcccc}
\hline & FFQ (\% FA) & 7-d ER (\% FA) & 1-d ER (\% FA) & 2-d ER (\% FA) \\
\hline PL (\% FAME) & 0.090 & $0.285^{\star}$ & $0.280^{\star}$ & $0.364^{*}$ \\
TG (\% FAME) & 0.124 & 0.272 & $0.409^{\star \star}$ & $0.361^{\star \star}$ \\
\hline
\end{tabular}

${ }^{*} P<0.05 ;{ }^{* *} P<0.01$.

† FAME - fatty acid methyl esters; \% FA - percentage of total daily fatty acid intake.

¥ 1-d ER = last day before blood sampling; 2-d ER = last two days before blood sampling. 
respectively ${ }^{15}$. Using a 3-day estimated record (3-d ER) mean intake of rumenic acid was 133 and $79 \mathrm{mg} \mathrm{day}^{-1}$ for men and women, respectively. Simultaneously, 3-day food duplicates were analysed revealing mean RA contents of 193 and $140 \mathrm{mg}$ day $^{-1}$ for men and women, respectively. The authors concluded that both dietary assessment methods might underestimate CLA intake. In comparison with the results of Ritzenthaler and coworkers $^{15}$, the calculated CLA intake values in the present study were much higher (Table 2), even higher than the data gained by food analysis. This might be due to differing nutritional habits (food choice) in both samples as well as to differences in the underlying CLA database. Furthermore, in the study of Ritzenthaler et $a l .{ }^{15}$ underreporting seems to be more prominent in women than in men, while in the present study distinct underreporters were excluded from the evaluation.

Regarding total fat intake in this study, different nutrient databases were used for the evaluation of the FFQ and the 7-d ER. The database for evaluation of the 7-d ER was Souci/Fachmann/Kraut (1986), which is known to overestimate total fat intake ${ }^{38}$; for the FFQ evaluation fat data from the German Federal Food Code nutrient database (version II.2) were applied. However, the similar differences given for rumenic acid and total fat intake between both methods, e.g. $24 \%$ and $23 \%$, do not hint to a major effect of the different databases used for total fat estimation. It has to be emphasised that the FFQ has been developed for CLA assessment and that rumenic acid evaluation was based on identical analytical data for both assessment tools.

The Pearson correlation coefficient is an indicator for the agreement of two assessment methods. The correlation coefficient is not influenced by systematic under- or overestimation of a method regarding the other one, but mainly by agreement of the two methods ${ }^{31}$. In the present study, significant correlation coefficients for both methods and both parameters - intake of rumenic acid and total fat - were found. Our results fit well with the correlation coefficients reported so far for the assessment of polyunsaturated fatty acid intake, which range between $r=0.22$ and $0.59^{32,33,35,36,39}$ (rumenic acid: $r=0.46$, Table 3), as well as for the assessment of total fat intake with $r=0.47^{32}$ ( $r=0.43$, Table 3$)$. Adjustment for energy intake $^{19}$ would have given more precise correlation coefficients. However, with the newly developed FFQ only rumenic acid and total fat can be determined, which makes energy adjustment of the FFQ results impossible.

A further possibility to compare the intake results obtained with use of both methods is to classify the intake values into categories. In the present study a classification into tertiles was chosen. The results demonstrate the extent to which both methods rank the same individual equally, e.g. how many subjects are classified as being in the same intake tertile, in the adjacent tertile or in the opposite tertile. Two validation studies reported similar results when subjects were classified into tertiles of intake of fat and polyunsaturated fatty acids ${ }^{35,40}$. Two further studies found a higher percentage of the study group in the same quintile and a smaller percentage in the extreme quintile for total fat, linoleic acid and polyunsaturated fatty acids ${ }^{32,33}$.

In the literature, the kappa statistic is used less frequently to statistically confirm the results of crossclassification. The problem with this procedure is the classification into categories (tertile, quartiles, quintiles). Due to a small sample size it was assumed that the classification into tertiles was appropriate, although a classification into quintiles would have been likewise possible. In a validity study 53 persons were divided into quintiles in order to examine their cross-classification ${ }^{36}$.

Total kappa values in similar validation studies ranged between $0.07^{40}$ and $0.60^{37}$ for fat intake, and between $0.05^{37}$ and $0.33^{40}$ for polyunsaturated fatty acid intake. In the present study, the total kappa value for total fat intake was 0.13; however, for rumenic acid intake the total kappa value of 0.31 was high enough to reach statistical significance and consequently indicated an acceptable degree of identical ranking of subjects by both assessment methods.

Regarding the results of the fatty acid analysis in plasma TG and PL, the mean proportion of rumenic acid in TG was about twice as high as in the PL fraction. This finding is confirmed in a study on breast-fed infants ${ }^{41}$. These infants revealed rumenic acid proportions of $0.4 \%$ in plasma TG and $0.2 \%$ in plasma PL fatty acids. The proportions of the main fatty acids in plasma TG and PL were in the range reported in other studies ${ }^{42-44}$.

Rumenic acid (\% FA) intake as calculated by means of the 7-d ER showed a relatively high correlation coefficient with its proportions in plasma PL and TG. This indicates that the habitual dietary intake seems to be an important source of rumenic acid in human plasma. This has also been confirmed by the work of Britton et al. ${ }^{18}$, who reported that the content of rumenic acid rose significantly from 12.3 to $18.8 \mu \mathrm{mol} \mathrm{l}^{-1}$ in PL after a diet rich in rumenic acid. On the other hand, a diet low in rumenic acid provoked a drop of its content in PL from 14.3 to $8.9 \mu \mathrm{mol} \mathrm{l}^{-1}$. In another study the effect of linoleic acid intake on the plasma content of rumenic acid was tested $^{44}$. It was demonstrated that intake of linoleic acid did not modify the rumenic acid plasma content. Besides dietary intake, endogenous conversion of 11-transoctadecenoic acid to rumenic acid by means of delta-9desaturase activity has been discussed ${ }^{45-47}$ and seems to contribute to human CLA supply ${ }^{48}$. Therefore, the relationship between dietary intake and plasma contents of rumenic acid is confounded by endogenous synthesis. However, no valid estimate for the extent of confounding can be given so far. Regarding the analysis of CLA, it is likely that minor CLA isomers other than rumenic acid contributed to the rumenic acid peak of the 
chromatogram. As shown by Yurawecz et al. ${ }^{49}$, variable amounts of the $\mathrm{t} 7 \mathrm{c}$-isomer of $\mathrm{C} 18: 2$ could not be separated from the rumenic acid peak by means of the chosen methodology.

Dietary intake as assessed by means of an FFQ is expected to reflect the long-term dietary habits or the habitual diet, whereas the results gained with the 7-d ER technique represent the diet of a medium-term period better. Therefore, it seems likely that the intake of rumenic acid calculated by the FFQ shows a higher correlation with the proportion of rumenic acid in PL than in TG. However, both coefficients were very low. On the other hand, with the more precise but medium-term dietary assessment method of 7-d ER, relatively high correlation coefficients between rumenic acid intake and its contents in plasma PL and TG were demonstrated (Table 5). The best correlation was obtained between the short-term dietary intake of the last day (1-d ER) or the last two days (2-d ER) before blood sampling (7-d ER) and the rumenic acid proportion in plasma TG. This fits very well with the knowledge of lipidologists that the plasma TG fatty acid pattern reflects the recent (short-term) intake whereas the phospholipid fatty acids can be seen as a biomarker for the medium-term intake of fatty acids. It has to be acknowledged that this result also confirms the higher precision of the 7-d ER compared with the FFQ with its 46 food items even more, since no significant correlation with plasma fatty acids could be obtained.

\section{Conclusion}

With respect to the results of the 7-d ER, the content of rumenic acid in plasma TG and PL represent candidate biomarkers of the short-term and medium-term intake of rumenic acid, respectively. The newly developed short FFQ failed to show significant correlations with these plasma biomarkers although both dietary assessment methods showed an acceptable degree of correspondence in dietary CLA intake measurement.

\section{References}

1 Sehat N, Yurawecz MP, Roach JA, Mossoba MM, Kramer JK, $\mathrm{Ku}$ Y. Silver-ion high-performance liquid chromatographic separation and identification of conjugated linoleic acid isomers. Lipids 1998; 33: 217-21.

2 Chin SF, Liu W, Storkson J, Ha YL, Pariza MW. Dietary sources of conjugated dienoic isomers of linoleic acid, a newly recognized class of anticarcinogens. J. Food Comp. Anal. 1992; 5: 185-97.

3 Kavanaugh CJ, Liu KL, Belury MA. Effect of dietary conjugated linoleic acid on phorbol ester-induced PGE2 production and hyperplasia in mouse epidermis. Nutr. Cancer 1999; 33: 132-8.

$4 \mathrm{Ha}$ YL, Storkson J, Pariza MW. Inhibition of benzo(a)pyrene-induced mouse forestomach neoplasia by conjugated dienoic derivates of linoleic acid. Cancer Res. 1990; 50 1097-101.
5 Ip C, Scimeca JA. Conjugated linoleic acid and linoleic acid are distinctive modulators of mammary carcinogenesis. Nutr. Cancer 1997; 27: 131-5.

6 Lee KN, Kritchevsky D, Pariza MW. Conjugated linoleic acid and atheriosclerosis in rabbits. Atherosclerosis 1994; 108: $19-25$.

7 Kritchevsky D, Tepper SA, Wright S, Tso P, Czarnecki SK. Influence of conjugated linoleic acid (CLA) on establishment and progression of atherosclerosis in rabbits. J. Am. Coll. Nutr. 2000; 19: 472S-7S.

8 Belury MA. Conjugated dienoic linoleate: a polyunsaturated fatty acid with unique chemoprotective properties. Nutr. Rev. 1995; 53: 83-9.

9 Park Y, Storkson JM, Albright KJ, Liu W, Pariza MW. Evidence that the trans-10,cis-12 isomer of conjugated linoleic acid induces body composition changes in mice. Lipids 1999; 34: 235-41.

10 Zambell KL, Keim NL, Van Loan MD, Gale B, Benito P, Kelley DS, Nelson GJ. Conjugated linoleic acid supplementation in humans: effects on body composition and energy expenditure. Lipids 2000; 35: 777-82.

11 Medina EA, Horn WF, Keim NL, Havel PJ, Benito P, Kelley DS, Nelson GJ, Erickson KL. Conjugated linoleic acid supplementation in humans: effects on circulating leptin concentrations and appetite. Lipids 2000; 35: $783-8$.

12 Kelley DS, Taylor PC, Rudolph IL, Benito P, Nelson GJ, Mackey BE, Erickson KL. Dietary conjugated linoleic acid did not alter immune status in young healthy women. Lipids 2000; 35: 1065-71.

13 Basu S, Smedman AE, Vessby B. Conjugated linoleic acid induces lipid oxidation in humans. FEBS Lett. 2000; 468: 33-6.

14 Fritsche J, Steinhart H. Amounts of conjugated linoleic acid (CLA) in German foods and evaluation of daily intake. $Z$. Lebens. Unters. Forsch. A 1998; 206: 77-82.

15 Ritzenthaler KL, McGuire MK, Falen R, Shultz TD, Dasgupta $\mathrm{N}$, McGuire MA. Estimation of conjugated linoleic acid intake by written dietary assessment methodologies underestimates actual intake evaluated by food duplicate methodology. J. Nutr. 2001; 131: 1548-54.

16 Huang Y-C, Luedecke LO, Shultz TD. Effect of Cheddar cheese consumption on plasma conjugated linoleic acid concentrations in men. Nutr. Res. 1994; 14: 373-86.

17 Jiang J, Wolk A, Vessby B. Relation between the intake of milk fat and the occurrence of conjugated linoleic acid in human adipose tissue. Am. J. Clin. Nutr. 1999; 70: $21-7$.

18 Britton M, Fong C, Wickens D, Yudkin J. Diet as a source of phospholipid esterified 9,11-octadecadienoic acid in humans. Clin. Sci. 1992; 83: 97-101.

19 Willett WC. Nutritional Epidemiology. New York: Oxford University Press, 1998.

20 Rohrmann S. Entwicklung und Validierung von Kurzfragenbögen zur Erfassung der Fettaufnahme. Hannover: Magisterarbeit des Ergänzungsstudiengangs Bevölkerungsmedizin und Gesundheitswesen, 1998.

21 Gedrich K. Ökonometrische Bestimmung der Lebensmittelund Näbrstoffzufubr von Personen anhand des Lebensmittelverbrauch von Haushalten. Frankfurt am Main: Lang, 1997.

22 Hofmann M, Lydtin H. Bayerisches Kochbuch. München: Berkenverlag, 1992.

23 Harris JA, Benedict FG. Biometric Studies of Basal Metabolism in Man. Washington, DC: Carnegie Institute of Washington, 1919.

24 Schofield WN. Predicting basal metabolic rate, new standards and review of previous work. Hum. Nutr. Clin. Nutr. 1985; 39: 5-41.

25 Folch J, Lees M, Sloane-Stanley GA. A simple method for 
isolation and purification of total lipids from animal tissue. J. Biol. Chem. 1957; 226: 497-509.

26 Stahl E. Dünnschichtchromatographie. Berlin: Springer Verlag, 1967.

27 Schulte E, Weber K. Schnelle Herstellung der Fettsäuremethylester aus Fetten mit Trimethylsulfoniumhydroxid oder Natriummethylat. Fat Sci. Technol. 1989; 5: 181-3.

28 Fleiss JL. The Measurement of Interrater Agreement. New York: John Wiley \& Sons, 1981.

29 Landis JR, Koch GG. The measurement of observer agreement for categorical data. Biometrics 1977; 33 159-74

30 Kemppainen $\mathrm{T}$, Rosendahl A, Nuutinen $\mathrm{O}$, Ebeling $\mathrm{T}$ Pietinen $\mathrm{P}$, Uusitupa M. Validation of a short dietary questionnaire and a qualitative fat index for the assessment of fat intake. Eur. J. Clin. Nutr. 1993; 47: 765-75.

31 Riboli E, Elmstahl S, Saracci R, Gullberg B, Lindgarde F. The Malmo Food Study: validity of two dietary assessment methods for measuring nutrient intake. Int. J. Epidemiol. 1997; 26(Suppl. 1): S161-73.

32 Decarli A, Franceschi S, Ferraroni M, Gnagnarella P, Parpinel MT, La Vecchia C, Negri E, Salvini S, Falcini F, Giacosa A. Validation of a food-frequency questionnaire to assess dietary intakes in cancer studies in Italy. Results for specific nutrients. Ann. Epidemiol. 1996; 6: 110-8.

33 Egami I, Wakai K, Kato K, Lin Y, Kawamura T, Tamakoshi A, Aoki R, Kojima M, Nakayama T, Wada M, Ohno Y. A simple food frequency questionnaire for Japanese diet - Part II. Reproducibility and validity for nutrient intakes. $J$. Epidemiol. 1999; 9: 227-34.

34 Curtis AE, Musgrave KO, Klimis-Tavantzis D. A food frequency questionnaire that rapidly and accurately assesses intake of fat, saturated fat, cholesterol, and energy. J. Am. Diet. Assoc. 1992; 92: 1517-9.

35 Bonifacj C, Gerber M, Scali J, Daures JP. Comparison of dietary assessment methods in a southern French population: use of weighed records, estimated-diet records and a food-frequency questionnaire. Eur. J. Clin. Nutr. 1997; 51 217-31.

36 Horwath CC. Validity of a short food frequency questionnaire for estimating nutrient intake in elderly people. $B r . J$. Nutr. 1993; 70: 3-14.

37 McPherson RS, Kohl HW, Garcia G, Nichaman MZ, Hanis CL. Food-frequency questionnaire validation among Mexican-Americans: Starr County, Texas. Ann. Epidemiol. 1995; 5: 378-85.

38 Linseisen J, Wolfram G. Unterschiede in der Nährstoffzufuhr bei Verwendung verschiedener Nährstoff-Datenbanken ein Fallbeispiel. Z. Ernäbrungswiss. 1997; 36: 127-32.

39 Bohlscheid-Thomas S, Hoting I, Boeing H, Wahrendorf J. Reproducibility and relative validity of energy and macronutrient intake of a food frequency questionnaire developed for the German part of the EPIC project. European Prospective Investigation into Cancer and Nutrition. Int. J. Epidemiol. 1997; 26(Suppl. 1): S71-81.

40 Winkler G. Validierung einer Food-Frequency-Erhebung. Dissertation, Institut für Sozialökonomie, Institut für Epidemiologie, Freising/Neuherberg, 1992.

41 Innis SM, King DJ. Trans fatty acids in human milk are inversely associated with concentrations of essential all-cis $\mathrm{n}-6$ and $\mathrm{n}-3$ fatty acids and determine trans, but not $\mathrm{n}-6$ and $\mathrm{n}-3$, fatty acids in plasma lipids of breast-fed infants. Am.J. Clin. Nutr. 1999; 70: 383-90.

42 Asciutti-Moura LS, Guilland JC, Fuchs F, Richard D, Klepping J. Fatty acid composition of serum lipids and its relation to diet in an elderly institutionalized population. Am. J. Clin. Nutr. 1988; 48: 980-7.

$43 \mathrm{Ma}$ J, Folsom AR, Eckfeldt JH, Lewis L, Chambless LE. Shortand long-term repeatability of fatty acid composition of human plasma phospholipids and cholesterol esters. The Atherosclerosis Risk in Communities (ARIC) Study Investigators. Am. J. Clin. Nutr. 1995; 62: 572-8.

44 Herbel BK, McGuire MK, McGuire MA, Shultz TD. Safflower oil consumption does not increase plasma conjugated linoleic acid concentrations in humans. Am. J. Clin. Nutr. 1998; 67: 332-7.

45 Mahfouz MM, Valicenti AJ, Holman RT. Desaturation of isomeric trans-octadecenoic acids by rat liver microsomes. Biochim. Biophys. Acta 1980; 618: 1-12.

46 Adlof RO, Duval S, Emken EA. Biosynthesis of conjugated linoleic acid in humans. Lipids 2000; 35: 131-5.

47 Griinari JM, Corl BA, Lacy SH, Chouinard PY, Nurmela KV, Bauman DE. Conjugated linoleic acid is synthesized endogenously in lactating dairy cows by delta(9)-desaturase. J. Nutr. 2000; 130: 2285-91.

48 Martin J-C, Banni S. Occurrence, chemistry and nutrition of conjugated linoleic acid. In: Sébédio JL, Christie WW, eds. Trans Fatty Acids in Human Nutrition. Dundee: The Oily Press, 1998; 261-302.

49 Yurawecz MP, Roach JA, Sehat N, Mossoba M, Kramer JK, Fritsche J, Steinhart H, Ku Y. A new conjugated linoleic acid isomer, 7 trans, 9 cis-octadecadienoic acid, in cow milk, cheese, beef and human milk and adipose tissue. Lipids 1998; 33: 803-9. 